\title{
Comparison of Efficiency of Video Endoscopy and Open Inguinal Lymph Node Dissection
}

\author{
SHUO WANG, PENG DU, XINGXING TANG, CHAO AN, NING ZHANG and YONG YANG \\ Key Laboratory of Carcinogenesis and Translational Research (Ministry of Education), \\ Urological Department, Peking University Cancer Hospital \& Institute, Beijing, P.R. China
}

\begin{abstract}
Aims: To compare the efficiency and complications of video endoscopy, inguinal lymphadenectomy and open inguinal lymph node dissection. Patients and Methods: Nineteen video endoscopy inguinal lymphadenectomies were performed on 16 patients from September 2014 to December 2015, and 21 open inguinal lymph node dissections were performed on 18 patients from September 2013 to September 2014. Surgical time, operative blood loss, incidence of complications, length of stay, lymph node harvest and suture removal time were compared between the two groups. Results: Operative time (135.5 \pm 45.52 vs. $169.8 \pm 55.19 \mathrm{~min})$, lymph node harvest $(10.78 \pm 5.22 \mathrm{vs}$. $12.60 \pm 5.53)$, and hospital stay (10.43 \pm 2.53 vs. $12.50 \pm 4.98$ days) did not statistically differ between the two groups $(p>0.05)$. However, operative blood loss $(22.50 \pm 14.24$ vs. $68.44 \pm 42.19$ $\mathrm{ml})$, drainage tube removal time $(7.23 \pm 1.79$ vs. $11.44 \pm 2.69$ days), incidence of complications $(21.05 \%$ vs. $61.9 \%)$, and suture removal time $(7.52 \pm 1.24$ vs. $12.81 \pm 2.04 \mathrm{~min})$ were significantly reduced in the video endoscopy surgery group compared with the open surgery group $(p<0.05)$. Conclusion: Compared with open surgery, video endoscopy surgery achieved the same efficiency, and had the advantage of reduction of complications, suture removal time and in-surgery blood loss.
\end{abstract}

Penile cancer is a rare disease, with an incidence rate of 0.1$0.9 / 100.000$ in Europe, 0.7-0.9/100.000 in America and 19/100.000 in Africa and Asia (1). The estimated number of new cases of penile cancer in the United States was 1250 for 2010, of which 310 died (2). The most common site of metastasis was the inguinal lymph nodes (ILNs); about $50 \%$ of patients presenting with palpable enlarged lymph nodes. Penile lymphatic drainage pathway can be divided into two groups superficial and deep. There are approximately 25 lymph nodes

Correspondence to: Peng Du, 52\# FuChengLu, Beijing, P.R. China. Tel: +86 13611376873, Fax: +86 1088196064, e-mail: dupeng9000@ 126.com

Key Words: Inguinal lymph node dissection, video endoscopy inguinal lymphadenectomy, complications. in the superficial area which can be divided into five zones superomedial, superolateral, inferomedial, inferolateral and central zone around the sapheno-femoral junction (3). The deep lymph nodes are deep to the fascia lata and medial to the femoral vein, comprising appoximately. Five nodes, with Cloquet's node being one of the deep nodes located between the femoral vein and lacunar ligament (4). Immediate surgical intervention has been shown to have a positive impact on survival in patients with lymph node involvement (5). The main surgical technique used is inguinal lymph node dissection (ILND). Open surgery used to be most commonly carried out, however, since Tobias-Machado et al. successfully performed the first ILND under video endoscopy (6), this technology has been widely used. At the Peking University Cancer Hospital and Institute, the first inguinal lymphadenectomy video endoscopy (VEIL) was performed in September 2014. From September 2014 to December 2015, we completed 19 VEIL procedures on 16 patients. Data were collected and compared with those from 21 open ILND which were performed on 18 patients from September 2013 to September 2014.

\section{Patients and Methods}

Study population. Our study included 34 patients with penile cancer treated at the Peking University Cancer Hospital and Institute. Penile amputation or radical resection was performed on all patients and the pathology diagnosed squamous cell carcinoma (SCC). Patients with distant metastasis were excluded. Age was between 28 to 69 years. All patients had a pelvic computed tomographic (CT) scan/magnetic resonance imaging (MRI), chest X-ray and electrocardiographic examination before surgery.

Surgical indication. Patients with one or more of the following indications were chosen for ILND - both superficial and deep lymph nodes were removed: i) Patients with palpable ILN, enlarged lymph nodes did not vanish or shrink after antibiotic treatment for 1 month; ii) enlarged inguinal metastases suspicious on preoperative imaging (CT or MRI); iii) clinical disease with intermediate or high risk for lymph node involvement according to National Comprehensive Cancer Network guidelines (7). [Pathology of resected penile samples was grade 2 or greater, or T2 (tumor invading corpus cavernosum urethral cavernosum) or greater]. 
From Sep 2014 to Sep 2015, we performed VEIL on patients with ILND indications, patients with skin lesions for tumor invasion at the operation site were excluded, and patients converted to open surgery were not included in this study. Synchronous pelvic lymph node dissection (PLND) was performed on patients with $\mathrm{N} 2$ or higher or if the diameter of the enlarged ILNs was greater than $4 \mathrm{~cm}$. Patients with coagulation defects, intake of non-steroid anti-inflammatory drugs in the previous week (e.g. aspirin), or other surgical contraindication (e.g. new onset of myocardial infarction) were excluded. No patients underwent chemotherapy or radiotherapy before surgery.

All 40 operations were performed by a single doctor at the Peking University Cancer Hospital and Institute, Six patients had bilateral ILND separately (three in the VEIL group, three in the open surgery group), while the other 28 patients underwent unilateral ILND (Table I).

Surgical technique. Open surgery group: Modified lymphadenectomy was performed (8) with the patient in a supine position. An inguinal incision was made commencing at approximately $2 \mathrm{~cm}$ superior to the inguinal arch, typically a $15-20 \mathrm{~cm}$ longitudinal skin incision. The superficial dissection was carried out above the level of the external oblique fascia and femoral lata femoris. The boundaries of the dissection were defined by the line of the anterior superior iliac spine and the external ring, laterally from the anterior superior iliac spine extending $20 \mathrm{~cm}$ inferiorly, with the media margin in the line of the pubic tubercle extending $15 \mathrm{~cm}$ downward. The saphenous vein and its five branches were carefully separated and ligated. Deep lymph node dissection was performed in all cases; the femoral artery and veins were exposed after cutting open the femoral canal sheath as lymph nodes needed to be dissected between vessels. In cases with two or more enlarged lymph nodes, PLND would be performed. The boundaries of PLND included the ilio-inguinal nerve for the lateral margin, obturator nerve for the medial margin, bifurcation of iliac vessels for the superior margin, arteria circumflexa ilium for the lateral margin and deep to the obturator nerve. The sartorius was mobilized as a flap from the top and covered the femoral vessels (9). In cases with a large residual cavity, a full-thickness suture was used to reduce the subcutaneous space, and a subcutaneous drainage tube was inserted or a second pelvic drainage tube was used in PLND cases.

VEIL group: The patient was placed in a supine position with the thighs apart at 30 degrees of abduction and external rotation. An incision of $1.5 \mathrm{~cm}$ in length was made $2 \mathrm{~cm}$ below the apex of the femoral triangle, blunt separation above the Scarpa fascia was performed by finger dissection, an air pocket was inserted into the space and $600 \mathrm{ml}$ gas was injected and maintained for $2 \mathrm{~min}$. Incisions of $0.5 \mathrm{~cm}$ (left hand) and $1 \mathrm{~cm}$ (right hand) length were made about $5 \mathrm{~cm}$ interior and lateral to the first incision site $(2 \mathrm{~cm}$ below the apex of the femoral triangle).

Two $12 \mathrm{~mm}$ trocars and one $5 \mathrm{~mm}$ trocar were placed through three incision sites. The position of the trocars was adjusted according to the patient's individual situation (Figure 1). Fifteen millimeters of pressure were maintained during surgery (Figure 2). The roof of the cavity was composed of skin and Camper's fascia, the base was composed of the superficial lymph node and femoral lata femoris. Surgical boundaries were the same as for open surgery. Firstly, superficial lymph node dissection was performed, the root of the saphenous vein and its five branches were carefully separated and ligated by clips, then the femoral canal sheath was opened, and the deep lymph nodes between the vessels were dissected. In some

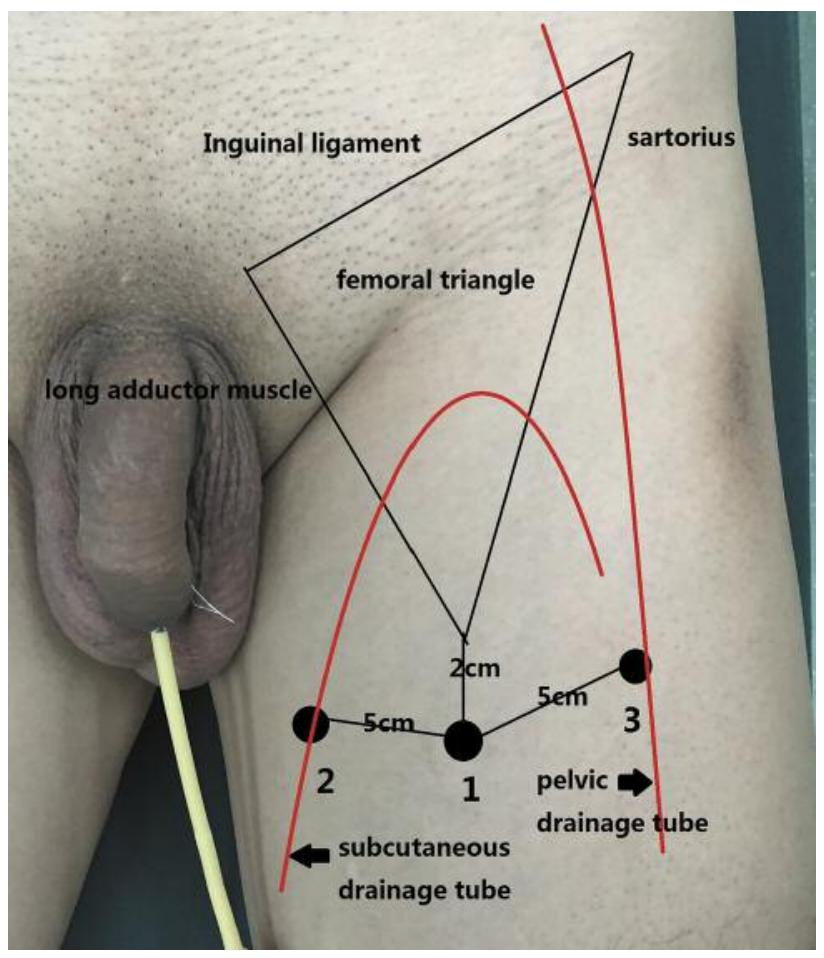

Figure 1. Patient's position and trocar placement during VEIL surgery. Camera lens is located at site 1; laparoscopic forceps located; subcutaneous drainage tube would be placed at site 2, pelvic drainage tube would be placed at site 3 .

cases requiring PLND, the inguinal ligament needed to be cut below the lateral margin of the peritoneum in order to reach the PLNs. The inguinal ligament was sutured under video endoscopy after PLN clearance. In cases with a massive tissue defect, 3-5 full-thickness sutures were performed to close the subcutaneous space, one subcutaneous drainage tube was used (Figure 3), or another pelvic drainage tube was used in patients undergoing PLND. All procedures were completed under video endoscopy.

Postoperative treatment. Four days of immobilization was required after surgery, with daily subcutaneous injection of $0.3 \mathrm{ml}$ low molecular weight heparin during this period for prevention of lower extremity phlebothrombosis. A 2-kg sandbag was placed above the wound and continuous negative pressure suction by drainage tube was necessary. Antibiotic medicine was given routinely for 4-7 days; patients with a wound infection were given antibiotics until the infection was controlled. Routinely, drainage tubes were removed when the drainage volume was less than $50 \mathrm{ml}$ for 2 days. Resection of necrotic skin, longer antibiotic treatment, continuous subcutaneous drainage or needle puncture drainage was given to patients with complications such as wound infection, skin necrosis, lymphocele or hematoma. In patients with delayed lymphocele or hematoma after drainage tube removal, needle aspiration was performed. Patients with clinical stage $\mathrm{cN} 2-\mathrm{N} 3$ were recommended to undergo 3-4 phases of bleomycin, cis platinum and etoposide (TIP) chemotherapy after surgery. 


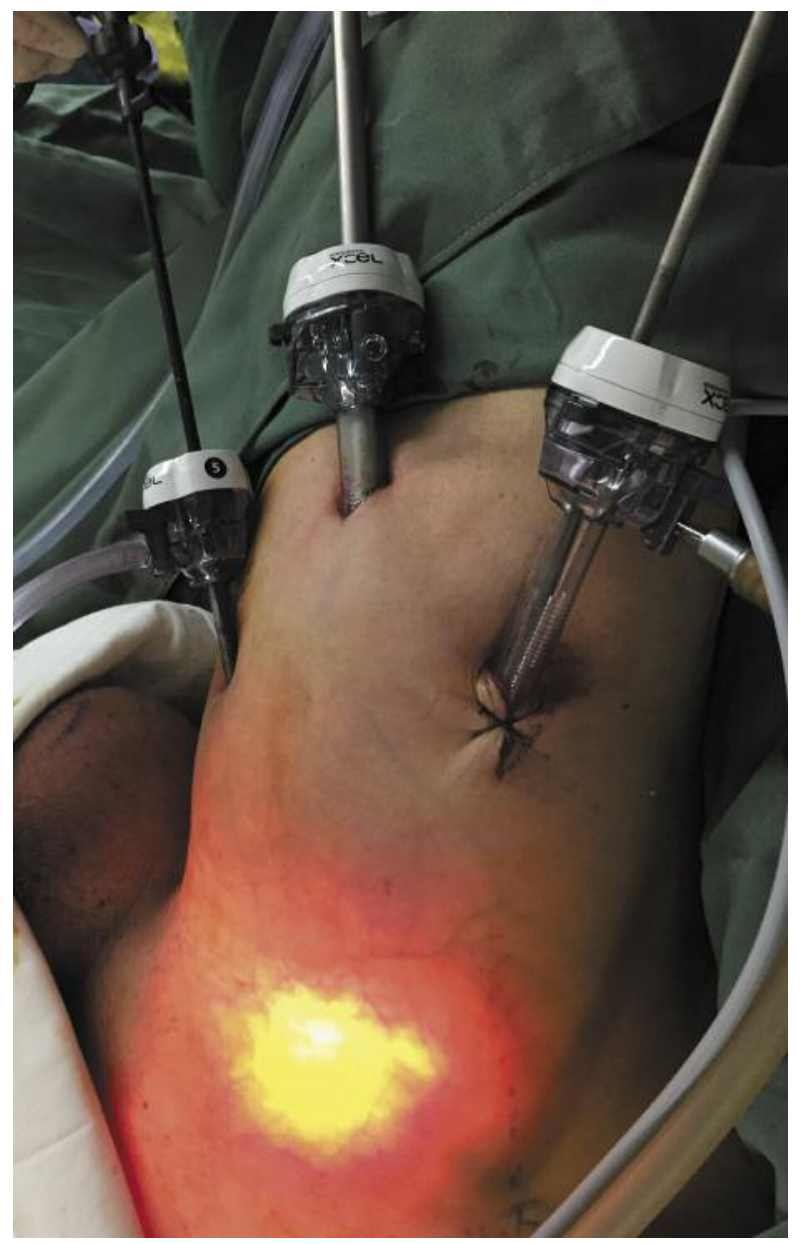

Figure 2. Right side inguinal lymph node dissection during VEIL.

Observation targets. Length of procedure, operative blood loss, lymph node harvest, drainage tube removal time, complications, length of hospital stay, pathology and suture removal time were analyzed and compared between the two groups. Clavien grading IIV was used for judging complications associated with surgery.

Statistical analysis. The data were analyzed using SPSS (Statistical Package for the Social Science, Version 19.0 SPSS) software. All data were analyzed by homogeneity test and normality test, $t$-test and chi-square test were used to analyze differentiation between the two groups. Statistical significance was reached when $p \leq 0.05$.

\section{Results}

Both groups were comparable regarding demographic data (Table I). Operative time, lymph node harvest, and length of hospital stay did not differ statistically between the VEIL group and the open surgery group $(p>0.05)$. However,

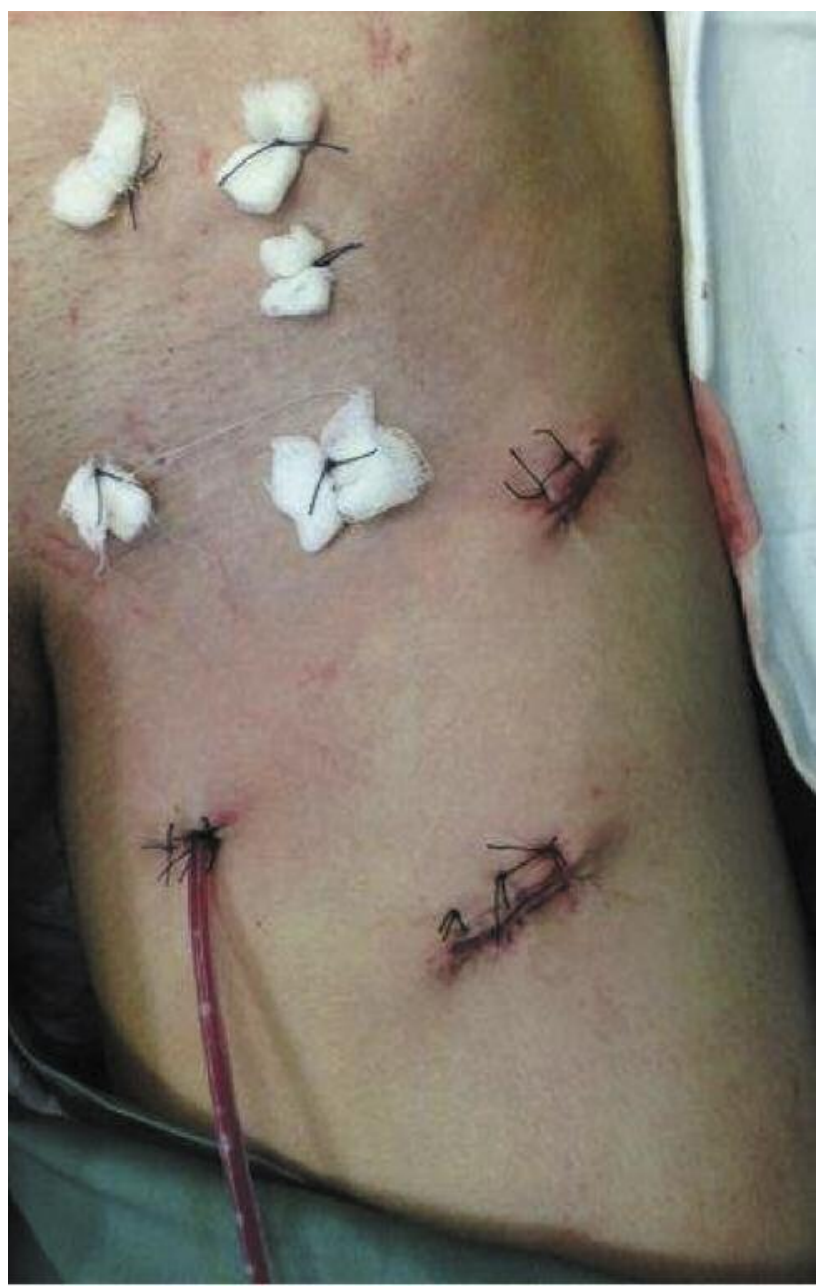

Figure 3. Subcutaneous drainage tube, wound and full-thickness suture.

operative blood loss, drainage tube removal time, complication rate and suture removal time were significantly reduced in the VEIL group compared with the open surgery group $(p<0.05)$ (Table II). Skin necrosis, wound infection and lymphedema were observed in both groups, but lymphocele and hematoma were observed only in the open surgery group (Table III).

Lymph node metastases were detected in 10 out of 16 patients in the VEIL group and 13 out of 18 patients in the open surgery group. The TNM staging of the patients in each group is shown in Table I.

Patients with cT2 or greater were recommended for TIP chemotherapy after surgery. Two patients in the VEIL group underwent three cycles of TIP chemotherapy, five patients in the open surgery group underwent 3-4 cycles of TIP chemotherapy and another patient with clinical stage N3 declined chemotherapy and other treatment. 
Table I. Patient's demographic and perioperative data.

\begin{tabular}{|c|c|c|c|}
\hline Characteristic & VEIL group (16 cases) & Open surgery group (18 cases) & p-Value \\
\hline Age & $53.57 \pm 9.87$ & $59.00 \pm 8.44$ & 0.06 \\
\hline Circumcised, n (\%) & $6 / 16(37.50 \%)$ & $6 / 18(33.3 \%)$ & 0.80 \\
\hline Maximum diameter of ILN (cm) & $1.66 \pm 0.67$ & $2.66 \pm 1.44$ & 0.03 \\
\hline Operative site: right/left, $\mathrm{n}$ & $8 / 11$ & $8 / 13$ & 0.80 \\
\hline Pathology confirmed LNs involved, n (\%) & $10 / 16(62.50)$ & $13 / 18(88.89)$ & 0.55 \\
\hline Clinical stage (cTNM), n (\%) & 16 & 18 & \\
\hline cTxNOMO & $6 / 16(37.5 \%)$ & $5 / 18(27.78 \%)$ & 0.55 \\
\hline cTxN1M0 & $8 / 16(50 \%)$ & $7 / 18(38.89 \%)$ & 0.52 \\
\hline cTxN2M0 & $1 / 16(6.25 \%)$ & $3 / 18(16.67 \%)$ & 0.35 \\
\hline cTxN3M0 & $1 / 16(6.25 \%)$ & $3 / 18(16.67 \%)$ & 0.35 \\
\hline Tumor recurrence in 12-month follow-up, n (\%) & $1 / 16(6.3 \%)$ & $2 / 18(11.1 \%)$ & 0.62 \\
\hline TIP chemotherapy after surgery, $\mathrm{n}(\%)$ & $2 / 16(12.5 \%)$ & $5 / 18(27.8 \%)$ & 0.27 \\
\hline
\end{tabular}

ILN, Inguinal lymph nodes; TIP, bleomycin, cis platinum and etoposide.

Table II. Surgical outcome of the two groups.

\begin{tabular}{|c|c|c|c|}
\hline Characteristic & VEIL group (19 cases) & Open surgery group (21 cases) & $p$-Value \\
\hline Mean \pm SD operative time, $\min$ & $139.5 \pm 45.52$ & $169.8 \pm 55.19$ & 0.08 \\
\hline Mean \pm SD intraoperative blood loss, $\mathrm{ml}$ & $22.50 \pm 14.24$ & $68.44 \pm 42.19$ & 0.00 \\
\hline PLND performed, $\mathrm{n}(\%)$ & $2 / 19(10.52)$ & $7 / 21(33.3)$ & 0.09 \\
\hline \multicolumn{4}{|l|}{ Mean \pm SD LN harvest } \\
\hline Total & $10.78 \pm 5.22$ & $12.60 \pm 5.53$ & 0.24 \\
\hline Superficial ILN & $8.71 \pm 5.72$ & $7.93 \pm 3.84$ & 0.60 \\
\hline Deep ILN & $2.22 \pm 1.86$ & $2.36 \pm 2.66$ & 0.89 \\
\hline PLN & $3.50 \pm 2.12$ & $4.0 \pm 2.07$ & 0.77 \\
\hline Mean \pm SD drainage tube removal time, days & $7.23 \pm 1.79$ & $11.44 \pm 2.69$ & 0.00 \\
\hline Mean \pm SD suture removal time, days & $7.58 \pm 1.24$ & $12.81 \pm 2.04$ & 0.00 \\
\hline Incidence of complications*, n (\%) & $4 / 19(21.05)$ & $13 / 21(61.90)$ & 0.01 \\
\hline Grade I & $3 / 19(15.79)$ & $8 / 21(38.10)$ & 0.12 \\
\hline Grade II & $1 / 19(5.26)$ & $5 / 21(23.81)$ & 0.10 \\
\hline Grade III & 0 & 0 & \\
\hline Grade IV & 0 & 0 & \\
\hline Mean \pm SD length of hospital stay, days & $10.43 \pm 2.53$ & $12.50 \pm 4.98$ & 0.15 \\
\hline \multicolumn{4}{|l|}{ Postoperative pathology, $\mathrm{n}(\%)$} \\
\hline G1, Well-differentiated & $7 / 19(78.95)$ & $9 / 21(66.67)$ & 0.70 \\
\hline G2, Moderately differentiated & $4 / 19(21.05)$ & $5 / 21(23.81)$ & 0.84 \\
\hline G3, Poorly differentiated & $0 / 19(0)$ & $2 / 21(9.5)$ & 0.17 \\
\hline
\end{tabular}

ILN, Inguinal lymph nodes; PLND, pelvic lymph node dissection. *Clavien.

In the VEIL group, the postoperative pathology (lymph nodes) in seven cases was G1 and four were G2, while in the open-surgery group, nine cases were $\mathrm{G} 1$, five cases were $\mathrm{G} 2$ and two were G3 (Table II).

Complications were observed in the two groups in the 12month follow-up: tumor recurrence occurred in three patients, two in the open surgery group (one underwent bilateral ILND with left-side tumor recurrence, the other underwent unilateral ILND) and one in the VEIL group (unilateral ILND performed); one further patient in the open surgery group underwent bilateral ILND but died after declining chemotherapy following tumor recurrence. The remaining two patients had undergone another three cycles of TIP chemotherapy and local three-dimensional optimal radiotherapy.

No lower extremity phlebothrombosis formation was seen in follow-up. Wound infection, skin necrosis, lymphedema were observed in both groups, but with antibiotic treatment, continuous subcutaneous drainage and aspiration and drainage all complications were controlled. 
Table III. Complications of the two groups [n (\%)].

\begin{tabular}{lccc}
\hline Complication & VEIL group (19 cases) & Open surgery group (21 cases) & $p$-Value \\
\hline Skin necrosis & $1 / 19(5.3 \%)$ & $6 / 21(28.6 \%)$ & 0.04 \\
Wound infection & $1 / 19(5.3 \%)$ & $3 / 21(14.29 \%)$ & 0.34 \\
Lymphedema & $3 / 19(15.8 \%)$ & $6 / 21(28.6 \%)$ & 0.33 \\
Lymphocele & $0 / 19(0 \%)$ & $5 / 21(23.8 \%)$ & 0.02 \\
Hematoma & $0 / 19(0 \%)$ & $1 / 21(4.8 \%)$ & 0.34 \\
Lower extremity phlebothrombosis & 0 & 0 & \\
\hline
\end{tabular}

\section{Discussion}

Tumors of the penis are the rarest tumors of the genitourinary system and represent $0.5 \%$ of malignant tumors in men. Squamous cell carcinoma accounts for more than $95 \%$ of cases of malignant disease of the penis (10). The most common metastatic pathway is to the ILNs followed by the PLNs. Identification of ILN involvement is important as a prognosticator of overall survival.

Open surgery was traditionally the most common procedure performed, with Daseler et al. first conducting open ILND in 1948 (3): An initial 20-cm incision was made at the lateral boundary of the ILND from the anterior superior iliac spine extending $20 \mathrm{~cm}$ inferiorly; this was followed by a $15 \mathrm{~cm}$ downward incision from the medial margin to the line of pubic tubercle and a $12 \mathrm{~cm}$ incision at the superior margin along the line of the external ring and superior iliac spine. The total resected area was approximately $187 \mathrm{~cm}^{2}$. In 1985, Fraley et $a l$. pioneered a modified ILND, preserving the great saphenous vein in order to reduce morbidity (11). ILND carried significant morbidity with high rates of both acute and chronic complications, with morbidity as high as $50 \%$ (12). The most commonly reported complications included skin necrosis $(0.6-$ $4.7 \%$ ), wound infections (1.2-1.4\%), lymphedema (5-13.9\%) and lymphocele formation (2.1-4\%) (13). In 1988, Catalona further improved the technique of ILND (8), the surgical area was limited to $99.8 \mathrm{~cm}^{2}$, the boundaries included the superior margin the line of external ring and superior iliac spine, the lateral margin from the anterior superior iliac spine extending $8 \mathrm{~cm}$ inferiorly and the medial margin to the line of pubic tubercle $9 \mathrm{~cm}$ downwards. This operation preserved the great saphenous vein and the tissue between the skin and Scarpa's fascia to reduce complications. At Peking University Cancer Hospital and Institute, we performed surgery according to the modified ILND, but we did not preserve the great saphenous vein in either group.

The literature was reviewed with particular emphasis on VEIL. Tobias-Machado was the first surgeon to successfully operate on one patient in 2006 using the VEIL procedure. In this patient, bilateral ILND was simultaneously performed on the left side by open surgery and on the right by VEIL, with skin necrosis occurring on the left side but none on the right (6). During 2008, Tobias-Machado et al. performed ILND on 30 patients ( 20 by VEIL and 10 by open surgery). Post-surgery data were compared between the two groups, showing that complications and in-hospital time were significantly reduced in patients undergoing VEIL. There was no tumor recurrence in any of the patients during the 31.9-month follow-up period, indicating that VEIL surgery was able to achieve the same tumor clearance effect as open surgery (14). Romanelli et al. reported the results of 33 VEIL and seven open surgery ILND procedures. The VEIL group operative time was $119 \mathrm{~min}$; the mean lymph node harvest was 8 ; the length of hospital stay was 5 days; complications occurred in 11 patients, including lymphocelce in nine patients; subcutaneous cellulitis occurred in two patients in the VEIL group, however, no skin necrosis occurred. All these results were better than those for the open group (15). Sotelo et al. carried out VEIL on eight patients with clinical stage T (2)N (0-3)M0. They found no woundrelated complications and lymphocele occurred in only three patients (16). Recently, with the development of robotic techniques, Sotelo et al. successfully presented bilateral robotassisted ILND on one patient, the patient present a left-side lymphocele on follow-up, with no other complications (17).

According to the literature, VEIL has been identified as an efficient, safe surgical technique with a low incidence of complications and has been widely used. However, the VEIL technique has only lately become an accepted procedure in China. Our research was one of the earliest studies; at Peking University Cancer Hospital and Institute, 40 ILND procedures have been performed on 34 patients in recent years, 19 by VEIL and 21 by open surgery. For patients requiring bilateral ILND, surgery was performed separately, bilateral ILND was not conducted simultaneously. Superficial and deep lymph node clearance was carried out in all cases, PLND was selectively carried out. There is controversy over whether PLND should be performed. At Peking University Cancer Hospital and Institute, PLND is performed only when PLN enlargement is present on CT/MRI examination, or when two or more enlarged lymph nodes are present, or when 
tumor diameter was found to be greater than $4 \mathrm{~cm}$ during surgery. When we compared data between the two groups, the results demonstrated that VEIL had the advantage of low hemorrhage, low incidence of complications and shorter suture removal time. The reasons for these advantages are: i) VEIL surgery significantly shortens incision length and thus reduces wound healing time; ii) the amplification effect of video endoscopy techniques allows small vessels and lymph vessels to be clearly observed and ligated, resulting in less hemorrhage and less lymphedema; iii) less skin tension may enhance wound healing.

However, there are still disadvantages of the VEIL procedure. With the laparoscopic lens placed $2 \mathrm{~cm}$ below the apex of the femoral triangle and two operating channels $5 \mathrm{~cm}$ interior and lateral to the apex of the femoral triangle, the surgeon sometimes needed to cut off the inguinal ligament in order to gain access to the pelvic region during PLND. The need to use two forceps to press down firmly to reach the surgical site is uncomfortable for the surgeon, leading to the possibility of residual lymph nodes due to their being missed at the bifurcation site of the iliac vessels as they would be difficult to reach. In future, we will try to place the laparoscopic lens and operating channels in the hypogastric region in order to complete the PLND and overcome these disadvantages.

\section{Conclusion}

VEIL has proven to be an efficient, radical and safe surgical method. Compared to traditional open surgery, VEIL in our study had minimal effects on the development of complications, and reduced in-surgery blood loss, in-hospital time and suture removal time. Our research reported 40 cases, however, more cases would be needed for further study. However, there were still disadvantages to the use of VEIL; this technique was hard to handle, a perfect surgical technique was required, and subcutaneous emphysema occurred in some patients. In patients with skin lesions near the tumor, VEIL surgery was not recommended because it was hard to place puncture channels and instruments appropriately and safely. Although we successfully completed surgery on patients with clinical stage N3, VEIL is not recommended especially for those with large tumor size. Severe local infiltration makes it difficult to create sufficient space, that would prolong the surgical time. In these cases, open surgery would be the first choice. Further studies to confirm the role and advantages of VEIL are needed.

\section{References}

1 Mobilio G and Ficarra V: Genital treatment of penile caicinoma. Curr Opin Urol 11: 299-304, 2001.

2 Jemal A, Siegal R and Miller KD: Cancer Statistics. CA Cancer J Clin 60: 277-300, 2010.
3 Daseler EH, Anson BJ and Reimann AF: Radical excision of the inguinal and iliac lymph glands; a study based upon 450 anatomical dissections and upon supportive clinical observations. Surg Gynecol Obstet 87: 679-694, 1948.

4 Loughlin KR: Surgical atlas. Surgical management of penile carcinoma: The inguinal nodes. BJU Int 97: 1125-1134, 2006.

5 McDougal WS: Preemptive lymphadenectomy markedly improves survival in patients with cancer of the penis who harbor occult metastases. J Urol 173: 681, 2005.

6 Tobias-Machado M, Tavares A, Molina WR Jr., Forseto PH, Juliano RV and Wroclawski ER: Video endoscopic inguinal lymphadenectomy (VEIL): minimally invasive resection of inguinal lymph nodes. Int Braz J Urol 32: 316-321, 2006.

7 National Comprehensive Cancer Network. (NCCN) Clinical Practice Guidelines in Oncology. Penile Cancer, Version 2.2017. Accessed 15 Apr 2017. https://www.nccn.org/professionals/ physician_gls/f_guidelines.asp.

8 Catalona WJ: Modified inguinal lymphadenectomy for carcinoma of the penis with preservation of saphenous veins: technique and preliminary results. J Urol 140: 306-310, 1988.

9 Sharp DS, Angermeier KW, Kavoussi L and Novick AC: Surgery of Penile and Urethral Carcinoma. In: Wein AJ: Campbell-Walsh Urology, Ninth Edition. Philadelphia: Saunders pp. 99-1022, 2007.

10 Mottet N, Avances C, Bastide C, Culine S, Ibora F, Kouri G, Ie Sourd A, Michel F, Pabot du, Chatelard P and Rocher L : Penile tumors (Tumeurs du penis). Prog Urol 14: 903-905, 2004.

11 Fraley EE, Zhang G and Sazama R: Cancer of the penis. Prognosis and treatment plans. Cancer 55: 1618-24, 1985.

12 Koifman L, Hampl D, Koifman N, Vides AJ and Ornellas AA: Radical open inguinal lymphadenectomy for penile carcinoma: surgical technique, early complications and late outcomes. J Urol 190: 2086-92, 2013.

13 Lughezzani G, Catanzaro M, Torelli T, Piva L, Biasoni D, Stagni S, Necchi A, Giannatempo P, Raggi D, Fare E, Colecchia M, Pizzocaro G, Salvioni R and Nicolai N: The relationship between lymph node ratio and cancer- specific survival in a contemporary series of patients with penile cancer and lymph node metastases. BJU Int 116: 727-723, 2015.

14 Tobias-Machado M, Tavares A, Silva MN, Molina WR, Forseto PH, Juliano RV and Wroclawski ER: Can video endoscopic inguinal lymphadenectomy achieve a lower morbidity than open lymph node dissection in penile cancer patients? J Endourol 22: 1687-1691, 2008.

15 Romanelli P, Nishimoto R, Suarez R, Nishimoto R, Suarez R, Decia R, Abreu D, Machado M, Arroyo C, Campolo H, Campos E, Carlos AS and Tobias-Machado M: Video endoscopic inguinal lymphandenectomy: surgical and oncological results. Actas Urologicas Espanolas (English Edition) 37: 305-310, 2013.

16 Sotelo R, Sanchez-Salas R, Carmona O, Garcia A, Mariano M, Neiva G, Trujillo G, Novoa J, Cornejo F and Finelli A: Endoscopic lymphadenectomy for penile carcinoma. J Endourol 21: 364-367, 2007.

17 Sotelo R, Cabrera M, Carmona O, de Andrade R, Martin O and Fernandez G: Robotic bilateral inguinal lymphadenectomy in penile cancer, development of a technique without robot repositioning: a case report. Ecancermedicalscience 26: 356, 2013.

Received May 19, 2017

Revised June 13, 2017

Accepted June 14, 2017 